\title{
Clinical utility of ulipristal acetate for the treatment of uterine fibroids: current evidence
}

\author{
This article was published in the following Dove Press journal: \\ International Journal of Women's Health \\ 26 March 2015 \\ Number of times this article has been viewed
}

\author{
Alice Trefoux Bourdet \\ Dominique Luton \\ Martin Koskas \\ Department of Obstetrics and \\ Gynecology, Bichat University \\ Hospital, Paris Diderot University, \\ Paris, France
}

\begin{abstract}
Uterine myoma is the most common benign uterine tumor in women of reproductive age and occurs in $20 \%-25 \%$ of the worldwide population. No currently approved medical treatment is able to completely eliminate fibroids. Surgery, particularly hysterectomy, predominates as the treatment strategy of choice, even though it is associated with risks and complications and causes infertility. Until recently, gonadotropin-releasing hormone agonists were the only available drugs for the preoperative treatment of fibroids. However, ulipristal acetate (UPA), an oral selective progesterone receptor modulator, was recently licensed in Europe for the same indication. Recent studies have demonstrated the efficacy and safety of UPA in the medical management of fibroids before surgery, with a better tolerability profile than leuprolide acetate. Analyzing the literature, we identified new management strategies involving UPA and surgery, considering advantages of both medical and surgical therapy. The advent of UPA will undoubtedly modify the surgical approach to fibroids, but the heterogeneity of these possible indications now requires various original clinical studies to identify the optimal indications for UPA in patients with symptomatic fibroid(s).
\end{abstract}

Keywords: uterine fibroids, medical treatment, selective progesterone receptor modulator, ulipristal acetate, surgery

\section{Introduction}

Uterine myoma (or leiomyoma or fibroid) is the main reason why women between 40 and 50 years old consult a gynecologist (apart from routine follow-up). It is the most common benign uterine tumor in women of reproductive age and occurs in $20 \%-25 \%$ of this population. ${ }^{1}$ Depending on the localization of the tumor, it may cause different symptoms including pelvic pain, dysmenorrhea, menorrhagia, iron deficiency anemia, infertility, and reduced quality of life.,3

No currently approved medical treatment is able to completely eliminate fibroids. For this reason, surgery, particularly hysterectomy, predominates as the treatment strategy. Other treatments such as myomectomy, hysteroscopic removal, and uterine artery embolization may also be proposed. The choice of treatment primarily depends on the symptoms, patient's age, desire to preserve fertility, and the patient's choice. ${ }^{4}$

Medical treatments are usually limited to preoperative reduction of symptoms, specifically heavy bleeding and anemia. Gonadotropin-releasing hormone ( $\mathrm{GnRH})$ agonists can be used as a presurgical treatment to create an artificial menopause state, ${ }^{5}$ thus reducing the size of the myoma and correcting anemia. However, this treatment is recommended only for short-term therapy because of the risks associated with artificially induced menopause, such as the loss of bone mineral density and the cardiovascular risk potentially related to long-term $\mathrm{GnRH}$ agonist therapy. ${ }^{6}$ Moreover, GnRH agonists are often poorly tolerated (mainly because of hot flashes).
Correspondence: Martin Koskas

Department of Obstetrics and Gynecology, Bichat University Hospital, 46 Rue Henri Huchard, 75018 Paris, France

Tel +33 | 40257673

Fax +33 I 40257696

Email martin.koskas@wanadoo.fr 
The use of oral progestin is also limited, because it may cause breakthrough and promote the proliferation of cells in the myoma. ${ }^{7,8}$ Moreover, the effects of progestin as a treatment for symptoms associated with fibroids have been poorly evaluated.

Because progesterone plays an important role in myoma growth, modulating the progesterone pathway represents one of the possibilities for medical therapy. Ulipristal acetate (UPA) is a selective progesterone receptor modulator (SPRM) that potently modulates the proapoptotic and proliferative activity of fibroid cells.

Following reports of the PEARL $\mathrm{I}^{9}$ and PEARL $\mathrm{II}^{10}$ studies in February 2012, UPA was licensed in Europe for preoperative fibroid treatment. More recently, the results of the first studies reporting long-term intermittent therapy (PEARL III, ${ }^{21}$ up to four courses of 3 months of UPA; PEARL IV, ${ }^{22}$ two courses of 3 months of UPA) have become available. UPA may become a new treatment option for many women who may wish to avoid surgery in the future. Our present review aims to evaluate the reported clinical utility of UPA and its place (both currently and in the future) in the treatment of uterine fibroids.

\section{Management of uterine fibroids: medical treatment's place?}

No currently available medical treatment is able to eliminate fibroids. Therefore, there is no indication for medical treatment in the absence of symptoms. ${ }^{11}$ In cases with symptomatic fibroids (pain or bleeding), the treatment has traditionally been surgical. Based on a large literature review, ${ }^{4}$ the French recommendations for the management of patients with fibroids concluded the following:

- When pregnancy is desired, the hysteroscopic resection of submucosal fibroids less than $4 \mathrm{~cm}$ in length is recommended.

- Interstitial, also known as intramural, fibroids have a negative effect on fertility, but treating them does not improve fertility. Myomectomy is therefore indicated only for symptomatic fibroids, depending on their size and number, and may be performed by laparoscopy or laparotomy.

- For perimenopausal women who have been informed of the alternatives and the risks, hysterectomy is the most effective treatment for symptomatic fibroids and is associated with a high rate of patient satisfaction.

Because uterine artery embolization is an effective treatment with low long-term morbidity, ${ }^{4}$ it is an option for symptomatic fibroids in women who do not want to become pregnant, and it is a validated alternative to myomectomy and hysterectomy that must be offered to patients.

Medical treatment can be used to control symptoms associated with fibroids and can allow surgery to be scheduled under better circumstances (eg, a higher hemoglobin level, or myoma size reduction). Danazol is also often used to control bleeding. However, no randomized controlled trial has proven benefits in the treatment of uterine fibroids. In addition, several side effects have been described, such as acne, hirsutism, weight gain, irritability, musculoskeletal pain, hot flashes, and breast atrophy, all of which limit its long-term use. ${ }^{12}$ Oral combined contraceptive pills are often used for young women to control menorrhagia and dysmenorrhea. Unfortunately, such therapy has been very poorly investigated in patients with symptomatic fibroids.

A levonorgestrel intrauterine device can provide a good reduction in menorrhagia, but its effect on the size of uterine fibroids is still being debated. ${ }^{13,14}$ Its use is not recommended in cases with severe distortion of the uterine cavity because of low probability of symptom improvement and the risk of expulsion. ${ }^{15}$

Before the arrival of SPRMs, the most efficient medical treatment, as both a conservative treatment and as a preoperative therapy, was GnRH agonists. They induce significant improvements of most symptoms related to fibroids (bleeding, anemia, and pain) and are able to reduce the volume of fibroids. ${ }^{5}$ However, these effects are transient, and the fibroids usually return to pre-therapy size within a few months of discontinuation. ${ }^{16}$ Furthermore, the chemical castration that GnRH agonists cause leads to menopausal symptoms that limit their long-term use.

Recently, SPRMs have been proved to be efficient in terms of reducing both the symptoms and size of fibroids. ${ }^{9,10}$ The efficacy of these drugs will likely diminish the role of surgery in the management of fibroids. UPA may permit less invasive surgery and might totally replace surgery in some cases.

\section{Mechanism of action of UPA on uterine fibroids}

The ratio of the estrogen receptor (ER) to progesterone receptor (PR) is higher in leiomyoma than in the corresponding normal myometrium, both in terms of the total cellular levels in each individual case and in the nuclear levels. ${ }^{17}$ It appears that estrogen sensitivity is higher in the leiomyoma than in the corresponding myometrium due to the relative increase in ER. The role of estrogen on myoma growth has been known for a long time. However, the roles of progesterone and PR 
have only recently been elucidated. Progesterone promotes fibroid growth in two ways: 1) it upregulates epidermal growth factor expression and $\mathrm{Bcl}-2$ protein expression; and 2) it downregulates the expression of the tumor necrosis factor gene. ${ }^{18}$

SPRMs are a new class of PR ligands that display tissueselective effects on target cells. UPA is an orally active synthetic SPRM that is characterized by a tissue-specific progesterone antagonist effect. ${ }^{19} \mathrm{UPA}$ reduces the proliferation of leiomyoma cells and induces apoptosis by increasing the expression of cleaved caspase- 3 and decreasing the expression of Bcl-2. Conversely, UPA downregulates the expression of angiogenic growth factors and their receptors. Thus, it inhibits neovascularization, cell proliferation, and survival in leiomyoma cells, but not in normal myometrial cells. ${ }^{20}$ UPA also has a central action on the hypothalamicpituitary-ovarian axis, and it inhibits or delays ovulation. However, UPA does not change the basic levels of luteinizing hormone or follicle-stimulating hormone, and the estradiol levels remain in the mid-follicular physiological range (60-150 pg/mL). Consequently, UPA does not lead to estrogen deficiency or the symptoms associated with estrogen deficiency. UPA induces amenorrhea in most women because of its interactions with endometrial PRs. ${ }^{19}$ However, it is important to keep in mind that UPA should not be given as (or instead of) a contraceptive, although it most likely reduces spontaneous fertility during treatment.

\section{Therapeutic efficacy of UPA in the treatment of uterine fibroids}

The clinical efficacy and tolerability profile of UPA have been tested in four randomized, double-blind, multi-national, Phase 3 trials. The first was PEARL I (PGL4001 versus placebo in uterine fibroids), which compared oral UPA (5 or $10 \mathrm{mg} /$ day) to placebo. ${ }^{9}$ The second was PEARL II (PGL4001 versus GnRH analog in uterine fibroids), which compared UPA (5-10 mg/day) with the GnRH analog, leuprolide acetate (LA) (one intramuscular injection per month of $3.75 \mathrm{mg}) .{ }^{10}$ The third was PEARL III (PGL4001 long-term treatment), where four courses were given over 3 months, with each course of UPA treatment separated by two menstrual cycles. ${ }^{21}$ The final study was PEARL IV, which evaluated the administration of two courses of 3 months of $10 \mathrm{mg} /$ day of $\mathrm{UPA}^{22}$ (Table 1).

\section{PEARL I}

In the first PEARL (PEARL I) study, 96 patients received UPA at $5 \mathrm{mg} /$ day, 98 received UPA at $10 \mathrm{mg} /$ day, and
48 women received placebo. ${ }^{9}$ Every woman received $80 \mathrm{mg}$ iron supplementation once daily during the active treatment phase. Treatment was started during the first 4 days of menstruation and was continued for 13 weeks.

The inclusion criteria were as follows: women between 18 and 50 years of age with fibroid-related menorrhagia and anemia and a myomatous uterus equivalent in size to a pregnant uterus of 16 weeks gestation or less, with at least one fibroid of $3 \mathrm{~cm}$ or more in diameter, but with no fibroid exceeding $10 \mathrm{~cm}$ diameter as determined by magnetic resonance imaging (MRI), were included. Fibroid-related menorrhagia was evaluated by the Pictorial Blood Assessment Chart (PBAC) score and was considered significant for inclusion when it was higher than 100 on days $1-8$ of menstruation. Similarly, fibroid-associated anemia was considered significant when the hemoglobin level was lower than $10.2 \mathrm{~g} / \mathrm{dL}$ without macrocytosis. ${ }^{9}$

The co-primary endpoints were the efficacy of UPA in controlling excessive bleeding (defined as a PBAC lower than 75) and the reduction of fibroid volume at week 13, as assessed by MRI. The secondary endpoints included bleeding pattern amenorrhea, changes in the hemoglobin, hematocrit, and ferritin values, pain, and quality of life. The tolerability of UPA, considering treatmentrelated adverse events and endometrial changes, was also assessed. ${ }^{9}$

The PEARL I trial demonstrated the effectiveness of UPA in controlling menorrhagia. ${ }^{9}$ At week 13 , bleeding was controlled in $91 \%, 92 \%$, and $19 \%$ of the women receiving UPA (5 mg), UPA (10 mg), and placebo, respectively. Bleeding control was achieved on day 8 in $75 \%$ of patients receiving 5 or $10 \mathrm{mg}$ UPA versus $6 \%$ who were administered placebo.

A significantly higher rate of patients in the UPA groups had a reduction in myoma volume and a uterine volume decrease of at least $25 \%$ at week 13 . In the subpopulation of patients who did not undergo surgery, the myoma volume reduction appeared to be maintained for 6 months after the end of treatment. Higher hemoglobin and hematocrit levels were observed in patients receiving UPA than in patients receiving placebo, though all patients received the same iron supplementation. The higher dose of UPA (10 mg) was more efficient for pain reduction than was the placebo ( -5.6 versus -2.5 ; pain was assessed with Short-Form McGill Pain Questionnaire). ${ }^{9}$ An important limitation of the PEARL I study was the limited duration of the study. In addition, the study focused on preoperative treatment but was not designed to evaluate possible treatment-related differences in surgical outcomes. 


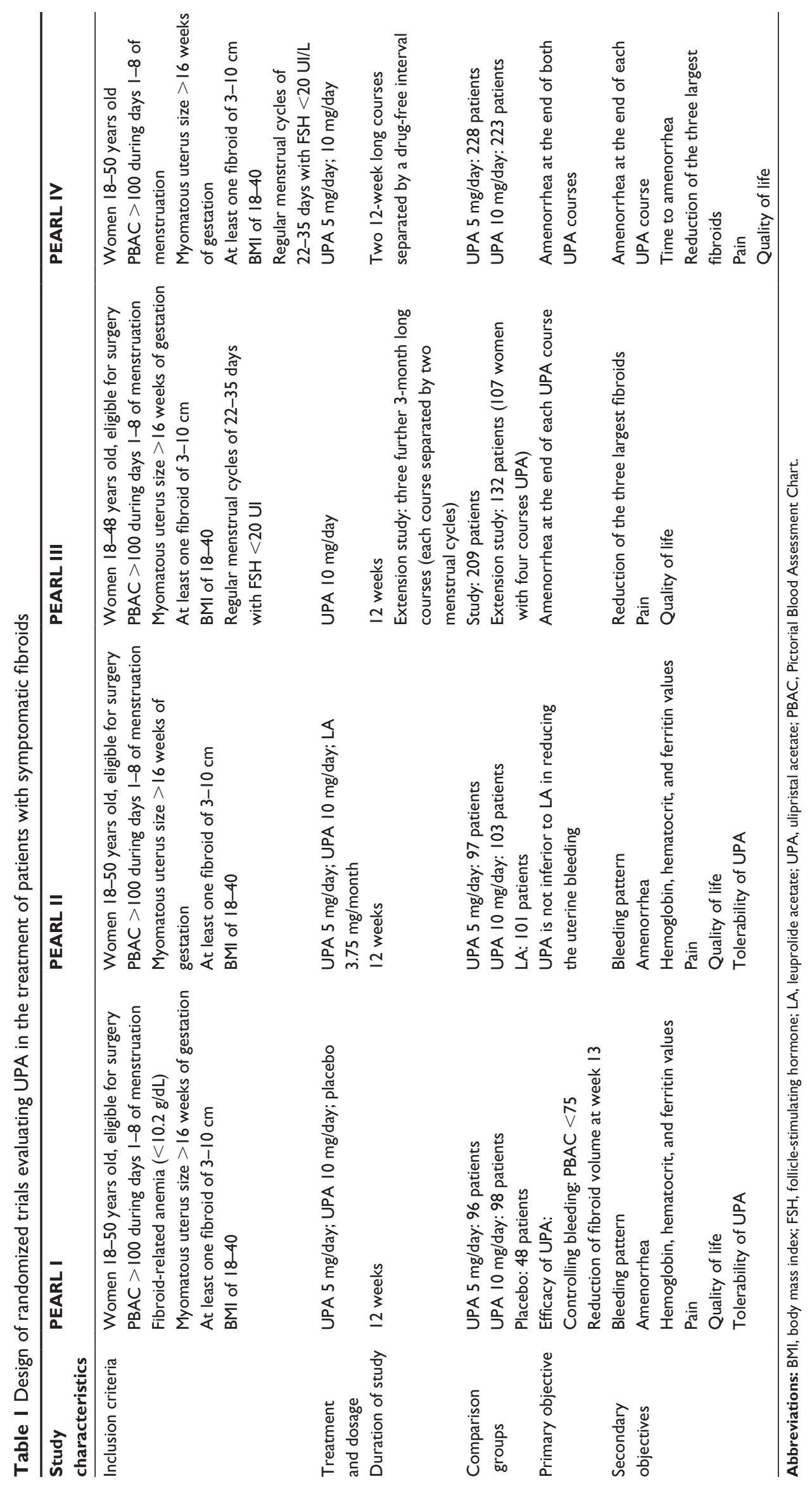




\section{PEARL II}

The PEARL II trial analyzed the efficacy and tolerability of UPA in patients with symptomatic fibroids by comparing it with LA, a GnRH agonist. Patients received UPA at 5 or $10 \mathrm{mg}$ once daily (97 and 103 women, respectively) or an intramuscular injection of $3.75 \mathrm{mg}$ LA (101 women) once per month. ${ }^{10}$

The inclusion criteria were the same as those in the PEARL I trial, except for anemia, which was not required. The primary endpoint was to demonstrate that UPA is not inferior to LA in reducing uterine bleeding. The secondary endpoints were similar to those of the PEARL I trial. ${ }^{9,10}$

Menorrhagia was controlled at week 13 in $90 \%, 98 \%$, and $89 \%$ of the women receiving UPA (5 mg), UPA (10 mg), and LA, respectively. ${ }^{10}$ Thus, UPA provided a quicker control of uterine bleeding compared with LA. The median time required to achieve amenorrhea was 7 days in the UPA group and 21 days in the LA group. All treatments reduced the volume of the three largest fibroids, with median reductions at week 13 of $36 \%$ in the group receiving $5 \mathrm{mg}$ of UPA, $42 \%$ in the group receiving $10 \mathrm{mg}$ of UPA, and $53 \%$ in the group receiving LA. LA was associated with a significantly greater reduction in uterine volume (47\%) than that observed in the UPA groups $(20 \%-22 \%)$. In the subpopulation of patients who did not undergo surgery, the myoma volume reduction appeared to be maintained for at least 6 months after the end of UPA treatment, whereas it returned to the baseline in most patients who had received LA. ${ }^{10}$ No significant difference in pain reduction between the three groups at the end of treatment was observed (pain scores of $-5.0,-6.0$, and -5.5 , respectively).

Finally, the trial aimed to prove the better tolerability profile of UPA in terms of the estradiol levels and incidence and intensity of hot flashes. ${ }^{10}$ At week 13 , the proportions of patients reporting moderate-to-severe hot flashes were significantly lower in the patients receiving UPA ( $11 \%$ in the $5 \mathrm{mg}$ group, and $10 \%$ in the $10 \mathrm{mg}$ group) than in the group of patients receiving LA (40\%) (Table 2).

These two trials (PEARL I and PEARL II) ${ }^{9,10}$ demonstrated an overall improvement in the quality of life of symptomatic women treated with UPA. Because the effects of daily administration of $5 \mathrm{mg}$ UPA did not differ significantly from the administration of $10 \mathrm{mg}, 5 \mathrm{mg}$ /day was defined as the minimum effective dose of the drug.

\section{PEARL III}

The PEARL III trial evaluated the efficacy of long-term intermittent use of $10 \mathrm{mg}$ /day of UPA. ${ }^{21}$ Women between

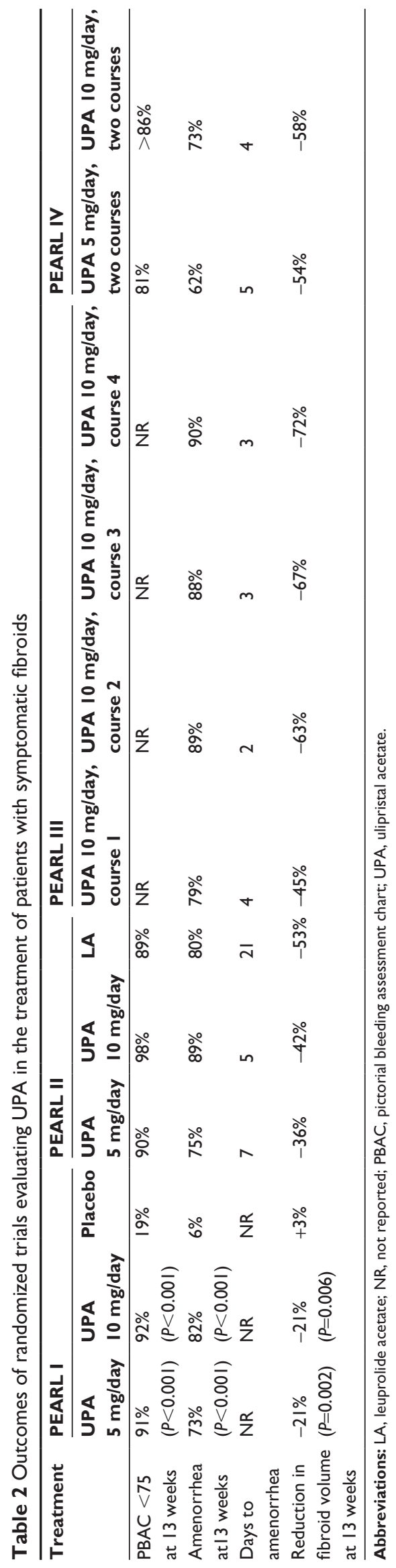


18 and 48 years of age with fibroid-related menorrhagia, a myomatous uterus (equivalent to 16 weeks' gestation or less), with at least one fibroid of $3 \mathrm{~cm}$ or more in diameter and none larger than $10 \mathrm{~cm}$, and who were eligible for surgery were included in the study.

Each patient received a 3-month course of $10 \mathrm{mg} /$ day of UPA, immediately followed by double-blind oral progestin norethisterone acetate (NETA; $10 \mathrm{mg} /$ day) or placebo for 10 days. UPA was started during the first 4 days of menstruation. After the first course of UPA, each woman could choose either to leave the study and attend a final follow-up visit at week 12, or to be assessed for a further 18 months and enroll in the PEARL III extension study to obtain three further 3-month long courses, with each course of UPA treatment separated by two menstrual cycles (approximately 2 months). A total of 209 patients were enrolled in the PEARL III trial; 132 entered the extension study, and 107 received four courses of UPA. ${ }^{21}$

After each course of UPA treatment, the control of bleeding (indicated by a significant reduction in the PBAC score) was faster than the initial reduction. The median times to amenorrhea were 2,3 , and 3 days for treatment courses 2,3 , and 4 , respectively. With every additional course, the amenorrhea rates increased. The amenorrhea rates were $89 \%$, $88 \%$, and $90 \%$ for the 131,119 , and 107 women who received treatment courses 2, 3, and 4, respectively. ${ }^{21}$

After each course, more patients demonstrated myoma volume reduction, and after four courses, $82 \%$ of patients had a $>25 \%$ reduction in the myoma volume. The median myoma volume decrease at each course was $-63 \%$ after course $2,-67 \%$ after course 3 , and $-72 \%$ after course 4 (Table 2). ${ }^{21}$

A number of limitations were associated with the PEARL III study. The dose (10 mg) and duration (3 months) of each UPA course were based on past experience, but it is unknown whether longer periods of continuous treatment could also prove safe and effective. Relatively few African women were recruited, but previous studies have demonstrated efficacy in these women. ${ }^{9}{ }^{10}$ Finally, one-third of the women (some of whom had surgery) were not enrolled in the extension study, and consequently, it is not possible to know how this subgroup of patients would have responded to repeated UPA.

\section{PEARL IV}

The PEARL IV study was designed to investigate the efficacy and safety of repeated 12-week courses of UPA for the intermittent treatment of symptomatic uterine fibroids. ${ }^{22}$ The inclusion criteria were similar to those detailed in the
PEARL III trial, but interestingly, to be included and randomized (to 5-10 mg daily of UPA), the patients did have to be scheduled for surgery. The second course of UPA was commenced with the second off-treatment menstruation. The proportion of patients achieving controlled bleeding during two treatment courses was $>80 \%$. Menstruation resumed after each treatment course and was diminished compared with baseline. After the second treatment course, the median reductions from baseline in the fibroid volume were $54 \%$ and $58 \%$ for the patients receiving 5 and $10 \mathrm{mg}$ of UPA, respectively. The pain and quality of life improved in both groups (Table 2). This study was conducted because the efficacy of long-term SPRM treatment in terms of bleeding control and fibroid shrinkage were unknown.

\section{Tolerability and safety profile Adverse events}

All PEARL trials have assessed the tolerability of UPA. The rate of the occurrence of any adverse event (Table 3) was approximately 50\% in the PEARL I trial (similar to the placebo group) and the PEARL IV trial (after the first course). Headache and pain, discomfort, or tenderness in the breasts were the most common adverse events in the UPA groups. Interestingly, this rate decreased during the second course of UPA in the PEARL IV trial. In the PEARL II trial, the rate of occurrence of any adverse event reached almost $80 \%$ in the UPA groups but exceeded $80 \%$ in the LA group.

The PEARL II trial focused on the incidence of hot flashes. Because this information was prospectively and specifically recorded, the incidence of hot flashes was higher in this trial. At week 13, UPA was associated with a significantly lower incidence of moderate-to-severe hot flashes compared with the LA group (11\% versus $40 \%$ ).

\section{Serious adverse events}

In the PEARL I trial, the incidence of serious adverse events (Table 3 ) was $2 \%$ in the groups treated with UPA $(6 \%$ in the placebo group), ${ }^{9}$ and similar rates were reported in PEARL II trial, with $8 \%, 5 \%$, and $6 \%$ of patients treated with UPA at $5 \mathrm{mg}$, UPA at $10 \mathrm{mg}$, and LA, respectively, experiencing serious adverse events. ${ }^{10}$

\section{Effects of UPA on the endometrium}

UPA has recently been introduced for the clinical management of premenopausal women. However, its powerful progesterone antagonist action could result in unopposed estrogen stimulation, thereby increasing the risk of hyperplasia and 


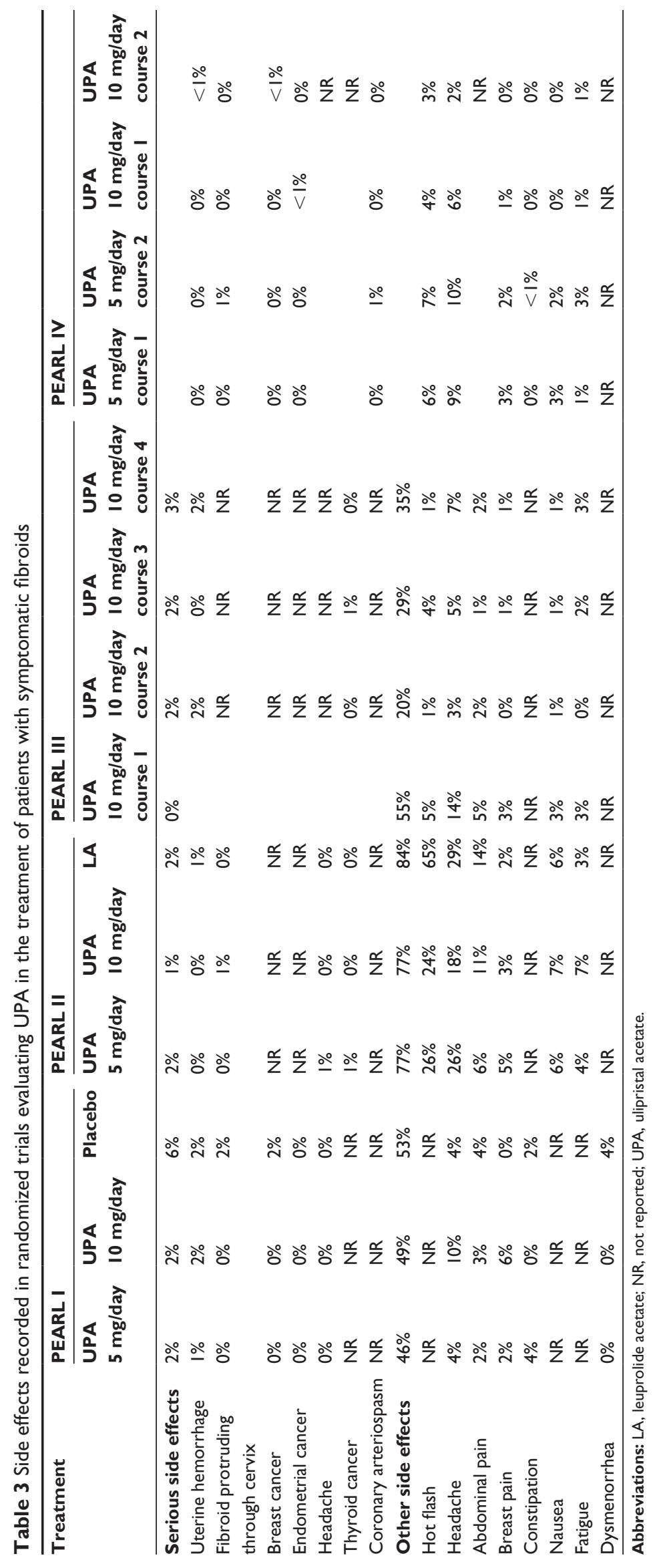


endometrioid carcinoma. These considerations inspired a workshop held in Bethesda, MD, USA in April 2006, where expert pathologists defined a common terminology and introduced specific recommendations for the interpretation of endometrial samples from patients treated with UPA. ${ }^{23}$ Various original aspects were described for these samples, but the expert pathologists did not observe malignant or premalignant changes. Some endometrial patterns resembled those observed during a normal endometrial cycle or other benign conditions, but some were unclassifiable based on the available criteria and were designated progesteron receptor modulators-associated endometrial changes (PAECs). Some of the aspects of PAECs are induced by hormonal depletion or by estrogen or progesterone stimulation.

In the PEARL I and II trials, endometrial biopsies allowed for detailed observations of the effects of UPA treatment on the endometrium. ${ }^{9,10}$ The histological modifications induced by UPA were compared with those detected in the two control groups (placebo and LA). Endometrial biopsies were repeated at three different times: at screening, after 3 months of treatment, and at 6 months after the end of therapy. After 3 months of treatment, PAECs were found in $50 \%-60 \%$ of women treated with UPA ( 5 or $10 \mathrm{mg}$ ) in the PEARL I and II trials. These changes had disappeared by 6 months after the end of treatment in both trials. The incidence of PAECs in both subgroups (5 and $10 \mathrm{mg}$ ) was comparable to that observed in the placebo and LA groups.

In the PEARL IV trial, an endometrial biopsy was performed 6 weeks after the end of the second course of UPA, and non-physiological features were observed in approximately $15 \%-20 \%$ of the patients. ${ }^{22}$ Thus, repeated courses of UPA did not increase the frequency of PAEC. The incidence of endometrial hyperplasia after two courses of treatment was $<1 \%$, which is consistent with the expected frequency in women with abnormal uterine bleeding within this age range. ${ }^{24}$ In addition, an increase in the mean endometrial thickness of treated patients has been observed on ultrasound examinations. In the PEARL I and II trials, an endometrial thickness exceeding $16 \mathrm{~mm}$ was reported in $3 \%-5 \%$ of cases at baseline and in up to $10 \%-15 \%$ of cases after 3 months of UPA administration. This phenomenon spontaneously regressed after the discontinuation of treatment (week 17). ${ }^{9,10}$ In conclusion, the typical changes induced by unopposed estrogen were not observed in patients treated with SPRM, and the risk of malignancy or premalignant endometrial tumors appeared to be low, even after repeated courses of UPA.

\section{UPA and pregnancy}

Recently, Luyckx et al reported the first series of pregnancies achieved after UPA treatment for uterine fibroids. ${ }^{25}$ In one of the institutions participating in the PEARL II and III trials, 21 of the 52 patients included in the study wished to conceive upon treatment completion. Among them, 19 underwent myomectomy after UPA, and two did not undergo any surgery. Two became pregnant without surgery, as their fibroids had regressed significantly and the uterine cavity was no longer distorted. Altogether, 15 patients conceived (71\%), totaling 18 pregnancies. Among these 18 pregnancies, 12 resulted in the birth of 13 healthy babies, and six pregnancies ended in early miscarriage (33\%). Among the six miscarriages, three occurred after IVF, which is known to have a higher miscarriage probability than natural conception. The others may have been related to the age of the population, whose median age was 38 years. The mean interval to achieve pregnancy after the end of the treatment was 10 months for patients starting directly upon treatment completion or after surgery. Patients who underwent surgery before trying to conceive were asked to wait 3 months to avoid early pregnancy on a scarred uterus.

In this study, there were no maternal complications related to fibroids during pregnancy or after delivery. There was no significant fibroid regrowth during pregnancy, possibly because of the apoptosis induced by UPA. Eleven of the 12 deliveries were performed by cesarean section, either because of previous surgery for uterine fibroids or in an emergency context for preeclampsia or fetal conditions. All of the infants were healthy. The only fetal anomaly observed occurred in a patient who started a new course of treatment when she was unexpectedly pregnant. This infant had an ectopic kidney, but the neonatology team concluded that the UPA treatment played no role in this anomaly. The pregnancies obtained in this series demonstrate that endometrial modifications are reversible and that the endometrium is suitable for implantation after treatment.

\section{Discussion}

In the treatment of fibroids, the choice of therapy is influenced by several factors, including the severity of symptoms (pain, bleeding), infertility related to fibroids, the tumor characteristics (volume, localization), the patient's age, wish to preserve the uterus, and wish to preserve fertility/desire to become pregnant.

The introduction of UPA will undoubtedly modify the use of surgery. Various situations can be cited: 
- Women with class 0,1 , or 2 symptomatic fibroids $>2 \mathrm{~cm}$ distorting the uterine cavity and wishing to preserve their fertility. With myoma reduction, UPA may make the hysteroscopic myomectomy procedure easier and reduce the number of two-step procedures.

- UPA could also become an alternative to hysteroscopic myomectomy if a good clinical response is obtained (improvement of menorrhagia) and if the uterine cavity is restored.

- Women with multiple symptomatic fibroids (class 2 to 5) wishing to preserve their fertility. UPA could be an alternative to myomectomy. Following laparotomic or laparoscopic myomectomy, pregnancy rates have reached $60 \%$, with good obstetric outcomes. ${ }^{26}$ Unfortunately, myomectomy is associated with surgical risks and complications (most importantly, blood loss requiring transfusion, long procedures and hospital stays, postoperative morbidity, and even life-threatening complications). ${ }^{27}$ Postoperative adhesions are of particular concern because their presence increases the risk of impaired spontaneous fertility. Avoiding laparotomy in young patients is most likely beneficial in terms of fertility, and the use of UPA could be an interesting alternative. Because the impact of such fibroids (not distorting the uterine cavity) on fertility is unclear, treating pain and bleeding symptoms first should be investigated. The main limitation of this strategy may be a matter of time. No data are available so far regarding the interval before fibroid regrowth and symptom recurrence after the end of UPA treatment. Waiting too long for spontaneous pregnancy or assisted reproductive technology attempts may be associated with a natural decline of the ovarian reserve. To date, the European Medicines Agency authorized UPA treatment for up to 3 months and stated that the 3-month treatment can be repeated, but only once. ${ }^{28}$

- Women with a few class 5 or 6 large myoma(s) could be treated with UPA preoperatively to permit laparoscopic myomectomy instead of laparotomy.

- Similarly, a sufficient uterine volume reduction permitting vaginal or laparoscopic hysterectomy instead of laparotomy may constitute a good reason to give UPA preoperatively.

- Perimenopausal women with one or more symptomatic fibroids who are unwilling to undergo surgery despite the failure of other medical treatments could be effectively treated with UPA while waiting for fibroids to shrink, as is usually observed after menopause. ${ }^{29}$
Currently, UPA is authorized for preoperative treatment for fibroids for a cycle of 3 months, which can be repeated once. For now, this treatment is not intended to avoid surgery, but in clinical practice, when fibroids symptoms have disappeared, the question to cancel or postpone surgery is frequently raised. Concerning the feasibility of myomectomy, on a technical point of view, it is particularly difficult to show "scientifically" that UPA facilitates or hampers the procedure.

The PEARL III and IV studies suggested the efficacy and safety of long-term intermittent treatment with UPA for the control of fibroid-related symptoms. ${ }^{21,22}$ The above list of possible new indications for medical therapy with UPA is interesting because it incorporates UPA into the current management of fibroids. Further studies are needed to confirm these new algorithms. Similarly, new algorithms should ideally consider identifying patients who will benefit from UPA therapy. To our knowledge, such identification has not yet been performed, but a multivariate analysis including the characteristics of the patients and fibroids associated with favorable clinical outcomes could permit the classification of patient as "expected good or bad UPA responders".

\section{Conclusion}

Currently, no validated medical treatment is able to eliminate fibroids, and hence, surgery still represents the most effective treatment for symptomatic fibroids. In many cases, hysterectomy is the treatment of choice, though it causes infertility in women of reproductive age. UPA may be a good option for women seeking pregnancy, for women who wish to avoid surgery, or before surgery to reduce the invasiveness of the operation. The heterogeneity of these possible indications will require various original clinical studies to identify the optimal indications for UPA in patients with symptomatic fibroid(s).

\section{Disclosure}

Dr Koskas is a member of the Scientific Advisory Board (SAB) of Gedeon Richter France since 2014. He did not receive a grant for this review. The other authors have no conflicts of interest. No financial support has been needed for this review.

\section{References}

1. Fernandez H, Chabbert-Buffet N, Koskas M, Nazac A. [Epidemiological data for uterine fibroids in France in 2010-2012 in medical center - analysis from the French DRG-based information system (PMSI)]. J Gynecol Obstet Biol Reprod (Paris). 2014;43(8):616-628. French. 
2. Fernandez H, Chabbert Buffet N, Allouche S. [Prevalence of uterine fibroids in France and impact on quality of life: results of a survey among 2500 women between 30-55 years]. J Gynecol Obstet Biol Reprod (Paris). 2014;43(9):721-727. French.

3. Donnez J, Jadoul P. What are the implications of myomas on fertility? A need for a debate? Hum Reprod. 2002;17(6):1424-1430.

4. Marret H, Fritel X, Ouldamer L, et al; CNGOF (French College of Gynecology and Obstetrics). Therapeutic management of uterine fibroid tumors: updated French guidelines. Eur J Obstet Gynecol Reprod Biol. 2012; 165(2):156-164.

5. Lethaby A, Vollenhoven B, Sowter M. Efficacy of pre-operative gonadotrophin hormone releasing analogues for women with uterine fibroids undergoing hysterectomy or myomectomy: a systematic review. BJOG. 2002;109(10):1097-1108.

6. Broekmans FJ. GnRH agonists and uterine leiomyomas. Hum Reprod. 1996;11(Suppl 3):3-25.

7. Nisolle M, Gillerot S, Casanas-Roux F, Squifflet J, Berliere M, Donnez J. Immunohistochemical study of the proliferation index, oestrogen receptors and progesterone receptors A and B in leiomyomata and normal myometrium during the menstrual cycle and under gonadotrophin-releasing hormone agonist therapy. Hum Reprod. 1999; 14(11):2844-2850.

8. Kim JJ, Sefton EC. The role of progesterone signaling in the pathogenesis of uterine leiomyoma. Mol Cell Endocrinol. 2012;358(2):223-231.

9. Donnez J, Tatarchuk TF, Bouchard P, et al; PEARL I Study Group. Ulipristal acetate versus placebo for fibroid treatment before surgery. N Engl J Med. 2012;366(5):409-420.

10. Donnez J, Tomaszewski J, Vázquez F, et al; PEARL II Study Group. Ulipristal acetate versus leuprolide acetate for uterine fibroids. $N$ Engl J Med. 2012;366(5):421-432.

11. Koskas M, Chabbert-Buffet N, Douvier S, Huchon C, Paganelli E, Derrien J. [Role of medical treatment for symptomatic leiomyoma management in premenopausal women]. J Gynecol Obstet Biol Reprod (Paris). 2011;40(8):858-874. French.

12. Ke LQ, Yang K, Li J, Li CM. Danazol for uterine fibroids [review]. Cochrane Database Syst Rev. 2009;3:CD007692.

13. Grigorieva V, Chen-Mok M, Tarasova M, Mikhailov A. Use of a levonorgestrel-releasing intrauterine system to treat bleeding related to uterine leiomyomas. Fertil Steril. 2003;79(5):1194-1198.

14. Murat Naki M, Tekcan C, Ozcan N, Cebi M. Levonorgestrel-releasing intrauterine device insertion ameliorates leiomyoma-dependent menorrhagia among women of reproductive age without a significant regression in the uterine and leiomyoma volumes. Fertil Steril. 2010;94(1):371-374.

15. Rizkalla HF, Higgins M, Kelehan P, O'Herlihy C. Pathological findings associated with the presence of a mirena intrauterine system at hysterectomy. Int J Gynecol Pathol. 2008;27(1):74-78.
16. Ortmann O, Diedrich K. Pituitary and extrapituitary actions of gonadotrophin-releasing hormone and its analogues. Hum Reprod. 1999; 14(Suppl 1):194-206.

17. Tamaya T, Fujimoto J, Okada H. Comparison of cellular levels of steroid receptors in uterine leiomyoma and myometrium. Acta Obstet Gynecol Scand. 1985;64(4):307-309.

18. Maruo T, Matsuo H, Shimomura Y, et al. Effects of progesterone on growth factor expression in human uterine leiomyoma. Steroids. 2003; 68(10-13):817-824.

19. Chabbert-Buffet N, Meduri G, Bouchard P, Spitz IM. Selective progesterone receptor modulators and progesterone antagonists: mechanisms of action and clinical applications. Hum Reprod Update. 2005; 11(3):293-307.

20. Chwalisz K, Perez MC, Demanno D, Winkel C, Schubert G, Elger W. Selective progesterone receptor modulator development and use in the treatment of leiomyomata and endometriosis. Endocr Rev. 2005;26(3):423-438.

21. Donnez J, Vázquez F, Tomaszewski J, et al; PEARL III and PEARL III Extension Study Group. Long-term treatment of uterine fibroids with ulipristal acetate. Fertil Steril. 2014;101(6):1565-1573.

22. Donnez J, Hudecek R, Donnez O, et al. Efficacy and safety of repeated use of ulipristal acetate in uterine fibroids. Fertil Steril. 2015; 103(2):519-527.

23. Williams AR, Bergeron C, Barlow DH, Ferenczy A. Endometrial morphology after treatment of uterine fibroids with the selective progesterone receptor modulator, ulipristal acetate. Int J Gynecol Pathol. 2012; 31(6):556-569.

24. Bonneau C, Perrin M, Koskas M, Genin AS, Rouzier R. [Epidemiology and risk factors for cancer of the uterus]. Rev Prat. 2014;64(6):774-779. French.

25. Luyckx M, Squifflet JL, Jadoul P, Votino R, Dolmans MM, Donnez J. First series of 18 pregnancies after ulipristal acetate treatment for uterine fibroids. Fertil Steril. 2014;102(5):1404-1409.

26. Goldberg J, Pereira L. Pregnancy outcomes following treatment for fibroids: uterine fibroid embolization versus laparoscopic myomectomy. Curr Opin Obstet Gynecol. 2006;18(4):402-406.

27. Uzan J, Koskas M, Fournier P, Margulies AL, Luton D, Yazbeck C. Colouterine fistula after polymyomectomy: a case report. J Med Case Rep. 2014;8:199.

28. European Medicines Agency; Science Medicines Health [homepage on the Internet]. Available from: http://www.ema.europa.eu/ema. Accessed January 21, 2015.

29. Polatti F1, Viazzo F, Colleoni R, Nappi RE. Uterine myoma in postmenopause: a comparison between two therapeutic schedules of HRT. Maturitas. 2000;37(1):27-32.
International Journal of Women's Health

\section{Publish your work in this journal}

The International Journal of Women's Health is an international, peerreviewed open-access journal publishing original research, reports, editorials, reviews and commentaries on all aspects of women's healthcare including gynecology, obstetrics, and breast cancer. The manuscript management system is completely online and includes

\section{Dovepress}

a very quick and fair peer-review system, which is all easy to use. Visit http://www.dovepress.com/testimonials.php to read real quotes from published authors. 